ISSN: 2600-5859

\title{
Evaluación del predio forestal San Vicente con fines de manejo y conservación ubicado en el cantón guano, Ecuador.
}

\section{Evaluation of the San Vicente forest plant for management and conservation purposes located in the cantón guano, Ecuador}

\author{
Hernán Eriberto Chamorro Sevilla. ${ }^{1}$, Gladys Janneth Urquizo Buenaño. ${ }^{2}$, Ana Lucila Cushpa \\ Guamán. ${ }^{3}$ \& David Francisco Lara Vásconez. ${ }^{4}$
}

\begin{abstract}
.
DOI: $\underline{\text { https://doi.org/10.33262/concienciadigital.v3i1.1.1137 }}$

This research is based on the management of the stands that make up the San Vicente forest estate, and is complemented by the description of its current situation and the calculation of dasometric indicators. These were required components for the generation of technical management strategies, through the application of systematic sampling when installing circular plots of $250 \mathrm{~m} 2$ for stand one, the census for stand 2 and exploratory sampling for stands 3, 4 and 5. According to the results, the San Vicente property is characterized by a stand formed by five blocks of Pinus radiata trees, stand two by Cupressus macrocarpa trees, stand 3 consists of two blocks of Eucalyptus globulus in the low latizal state, stand four or conservation zone formed by tree, shrub and herbaceous species and a fifth stand or area used. According to the above, it is proposed to apply thinning at an intensity of 20 to $30 \%$ for the blocks of stand one, pruning activity to the Cupresus macrocarpa trees of stand two, stand 3 requires gradual management of Eucalyptus trees globulus in a low latizal state; for sawn timber and posts, it could leave between 1-3 shoots per stump, and for fuel (firewood) a number greater than three; the stand four will be left as is and that the factors of the site affect until the next monitoring between 2-4 years. Finally, for stand five, it is proposed to reforest with the use of Pinus radiata seedlings at a distance of $3 \mathrm{~m} \times 3 \mathrm{~m}$ in order to re-establish the land use system, promoting the generation of ecological, economic and social benefits when
\end{abstract}

\footnotetext{
${ }^{1}$ Escuela Superior Politécnica de Chimborazo, Riobamba Ecuador, hernan.chamorro@espoch.edu.ec

${ }^{2}$ Escuela Superior Politécnica de Chimborazo, Riobamba Ecuador, gladys.urquizo@espoch.edu.ec

${ }^{3}$ Escuela Superior Politécnica de Chimborazo, Riobamba Ecuador, lucila.cushpa@espoch.edu.ec

${ }^{4}$ Diósesis de Riobamba, Riobamba Ecuador, davidlaravasconez@ hotmail.com
} 
ISSN: 2600-5859

applying criteria of sustainable management for the management of the forest resources of the evaluated property.

Keywords: Forest Zoning, Dasometry, Forest Species, St. Vincent Forest, Sustainable Management

\section{Resumen.}

La presente investigación se basa en la ordenación de los rodales que integran el predio forestal San Vicente, y es complementada por la descripción de su situación actual y el cálculo de indicadores dasométricos. Estos fueron componentes requeridos para la generación de estrategias de manejo técnico, mediante la aplicación del muestreo sistemático al instalar parcelas circulares de $250 \mathrm{~m}^{2}$ para el rodal uno, el censo al rodal 2 y el muestreo exploratorio a los rodales 3, 4 y 5 . Según los resultados, el predio San Vicente está caracterizado por un rodal formado por cinco bloques de árboles de Pinus radiata, el rodal dos por árboles de Cupressus macrocarpa, el rodal 3 consta de dos bloques de Eucalyptus globulus en estado latizal bajo, el rodal cuatro o zona de conservación formado por especies arbóreas, arbustivas y herbáceas y un quinto rodal o zona aprovechada. De acuerdo a lo expuesto, se propone aplicar raleo a una intensidad del 20 al $30 \%$ para los bloques del rodal uno, actividad de poda a los árboles de Cupresus macrocarpa del rodal dos, el rodal 3 requiere un manejo gradual de los árboles de Eucalyptus globulus en estado de latizal bajo; con fines de madera aserrada y postes se podría dejar entre 1-3 rebrotes por tocón, y para combustible (leña) un número mayor a tres; el rodal cuatro, se dejará como está y que los factores del sitio incidan hasta el próximo monitoreo entre 2-4 años. Finalmente, para el rodal cinco, se plantea reforestar con el uso de plántulas de Pinus radiata a un distanciamiento de $3 \mathrm{~m} \mathrm{x}$ $3 \mathrm{~m}$ a fin de establecer nuevamente el sistema de uso del suelo, propiciando la generación de beneficios ecológicos, económicos y sociales al aplicar criterios de manejo sustentable para la gestión de los recursos forestales del predio evaluado.

Palabras claves: Zonificación forestal, Dasometría, Especies Forestales, Bosque San Vicente, Manejo Sostenible

\section{Introducción.}

Los bosques constituyen una de las principales fuentes de vida para más de mil millones de personas, alojando a más de las tres cuartas partes de la biodiversidad terrestre mundial, y contribuyen al mismo tiempo con el desarrollo socioeconómico de cientos de millones de moradores de las zonas rurales con el aporte de numerosos productos y servicios como alimentos, medicinas y combustibles (Graziano da Silva, 2018).

En Ecuador el 34,7 \% de la superficie nacional está cubierta por bosques, siendo el 98.5\% bosques naturales, y las plantaciones el 1,5\% restante del patrimonio forestal (Grijalva et al., 2012). Debido 
a la influencia del sector forestal en la economía ecuatoriana, es prioritario disponer de información sobre los parámetros de crecimiento y productividad de los rodales para entender el funcionamiento del ecosistema y con ello generar estrategias de manejo técnico para contribuir con materia prima de calidad y elevar su productividad para satisfacer las necesidades del mercado nacional e internacional (Castellanos Bolaños et al., 2008).

El monitoreo de los recursos forestales consiste en la recolección sistemática de datos de las especies arbóreas y arbustivas de una zona determinada. Este permite la evaluación del estado actual, y principalmente sienta las bases de un proceso de planificación y ejecución de prácticas para la administración, uso y manejo de plantaciones u otros terrenos arbolados (Murrieta, Bryan, Delgado, Villalobos, \& Campos, 2007).

Este monitoreo ha demostrado que el deterioro de las áreas naturales a nivel mundial se ha incrementado sustancialmente, y uno de los factores de mayor influencia son las acciones humanas que aceleran dichas transformaciones (Alvarado \& Ospina, 2015). El Ecuador forma parte de los diecisiete países más diversos del mundo, con una cobertura boscosa en más de la mitad de su territorio, dentro de la cual existen zonas aptas para el aprovechamiento forestal. No obstante, no está exento de la presión que ejerce la actividad económica sobre su ecosistema (Sanchez, 2015). La zonificación territorial, desde un punto de vista ecológico y productivo, permite la identificación y delimitación de las principales zonas de importancia para la conservación de los recursos naturales (Collazo, Panizza, \& Paruelo, 2013).

En la actualidad existen herramientas tecnológicas, como los sistemas de información geográfica (SIG) y la percepción remota, que permiten optimizar el manejo de las zonas aptas para el aprovechamiento forestal en las que se pueden almacenar, analizar y vincular espacialmente las diferentes fuentes de información territorial que provengan de diversos sectores (Torres \& Peña, 2011).

Oyola Victor (2014), describe en su Libro sobre SIG, que la gestión de manejo de una masa forestal, implica algunas actividades como las siguientes, en las cuales se utiliza en mayor o menor medida información georreferenciada. Delimitación de las distintas zonas inventariables y unidades dasocráticas (montes, zonas aprovechadas, rodales, etc.), diseño de inventarios, realización de inventarios y gestión de sus datos para la obtención de resultados tales como estimaciones de volúmenes maderables. Gestión de infraestructuras del monte tales como vías de comunicación, torres de vigilancia contra incendios, etc.

En un contexto en el que no existen medios informáticos para la realización de estas tareas, gran parte de ellas se desarrollarán con el apoyo de cartografía clásica. Así, las zonas inventariables se delimitarán sobre un plano, y sobre este mismo pueden medirse sus superficies con la ayuda de un planímetro. En ese mismo plano se localizan las parcelas a muestrear en un inventario, y los operarios encargados de llegar hasta esas parcelas y realizar las mediciones pertinentes se ayudan 
ISSN: 2600-5859

de él para localizarlas y desplazarse sobre el terreno. Los resultados del inventario se almacenan en estadillos, y las operaciones correspondientes al análisis estadístico de estos se realizan de forma manual, así como la comparación con inventarios anteriores que permiten estudiar la evolución del monte (Oyola, 2014).

Reitera Oyola, que la presencia de estos medios facilita estas tareas, mejorando la gestión de los datos y las operaciones que pueden realizarse sobre estos. Un SIG es una herramienta que realiza tareas de uso y manejo de información geográfica, pues ha sido diseñada específicamente para trabajar con este tipo particular de información.

Sin embargo, enfatiza que el concepto de SIG ha evolucionado hasta convertir actualmente a estos en sistemas complejos que buscan dar solución a todas las necesidades que se presentan en situaciones similares a la de inventarios forestales. Con la tecnología actual, la incorporación de elementos propios de los SIG puede llegar mucho más allá, y uno de los pilares más sólidos de los SIG en la actualidad es su capacidad de mostrar que existe una componente espacial susceptible de ser gestionada con la ayuda de un SIG en la práctica totalidad de contextos posibles (Oyola, 2014).

Señala además que un SIG es una herramienta que brinda a las labores de uso y manejo de información geográfica toda la potencia de un ordenador, pues ha sido diseñada específicamente para trabajar con este tipo particular de información. No obstante, más allá de todas estas tareas antes mencionadas el concepto de SIG ha evolucionado hasta convertir actualmente a estos en sistemas complejos que buscan dar solución a todas las necesidades que se presentan en situaciones similares a las antes mencionadas. Con la tecnología actual, la incorporación de elementos propios de los SIG puede llegar mucho más allá, y uno de los pilares más sólidos de los SIG en la actualidad es su capacidad de mostrar que existe un componente espacial susceptible de ser gestionado con la ayuda de un SIG (Oyola, 2014).

Como sistema, un SIG puede gestionar la cartografía necesaria para la gestión integral del monte, y hacerlo además de forma centralizada. De este modo, se garantiza el rigor y la robustez de los datos base, ya que el SIG es el encargado de canalizar la utilización de estos por parte de todos los usuarios. Esto es de especial importancia en caso de que se editen los datos, ya que esta edición también está centralizada, y un usuario ve reflejarse en su cartografía de forma inmediata los cambios realizados por otro, teniendo siempre a su disposición la versión más actual y, por tanto, más adecuada (Oyola, 2014).

A esto puede añadirse la utilización de SIG móviles en dispositivos portátiles, que permiten que el SIG se incorpore también a las fases de trabajo de campo. Esa

misma cartografía centralizada pueden utilizarla los operarios en campo a través de sus dispositivos para desarrollar su trabajo, ayudándose además de sistemas de navegación para la 
ISSN: 2600-5859

localización de las parcelas de un muestreo o de cualquier otro punto de interés al que deban desplazarse (Oyola, 2014).

Gracias a los SIG, la información espacial puede ser aprovechada en mayor medida, y en muchos casos pasa de ser una información inherente a los datos, pero sin una verdadera aplicación, a ser un elemento sumamente enriquecedor y clave para muchos análisis (Oyola, 2014).

En el caso gestión forestal, los propios datos del inventario, que antes eran fundamentalmente datos sobre las propiedades de los distintos árboles medidos (altura, diámetro, etc.), ahora ofrecen muchas más posibilidades si se considera que cada uno de estos árboles ha sido medido en una parcela dada, la cual lleva asociadas unas coordenadas concretas (Oyola, 2014).

La poca información técnica disponible sobre los elementos que integran el predio forestal San Vicente para orientar su manejo considerando el uso actual al poseer áreas de terreno conformadas por especies arbóreas, arbustivas y herbáceas con potencial ecológico y comercial, son las razones que sustentan el desarrollo de la presente investigación, razón por la cual se plantearon como objetivos en primer lugar la necesidad de elaborar la distribución de los rodales que conforman el predio forestal San Vicente y en segundo lugar describir la situación actual, el cálculo de indicadores dasométricos para los rodales que posee el predio y la generación de estrategias de manejo técnico que orienten la preservación y manejo de estos recursos (J. Jiménez, Aguirre, \& Kramer, 2001).

\section{Metodología}

La investigación se realizó en el predio "San Vicente de la Curia Diocesana de Riobamba", situado en el barrio Pulug perteneciente a la parroquia San Isidro del cantón Guano, cuyas coordenadas geográficas son $78^{\circ} 42^{\prime} 23^{\prime \prime} \mathrm{O} 1^{\circ} 32^{\prime} 54^{\prime \prime} \mathrm{S}$ y que se encuentra ubicada a 3137 - $3146 \mathrm{msnm}$. La precipitación anual varía de 800 a $600 \mathrm{~mm}$, con una temperatura que varía desde los $2^{\circ} \mathrm{C}$ hasta los $13^{\circ} \mathrm{C}$, y se categoriza dentro de la formación: Bosque Siempre Verde Montano Alto de los Andes Orientales (Ordóñez, Rivera Seria, Ordóñez, Espinosa, \& Rivera P, 2004). El predio tiene una superficie total de 52,2 ha, y sus suelos son Inceptisoles o Entisoles, los cuales se caracterizan por su estado de desarrollo no avanzado y poca fertilidad (Fernández, 2015).

La investigación fue de tipo: documental, exploratoria, descriptiva, explicativa y de campo (Calvache, 2016) y se realizó en el segundo semestre del año 2017.

\section{Delimitación de usos del predio}

Tanto para el cálculo del área y perímetro de la zona de estudio, así como para la recolección de datos geográficos necesarios para la investigación, se realizó un recorrido de campo, mediante el cual se tomaron puntos geográficos usando un GPS (Global Positioning System), modelo Garmin 62Csc. Utilizando sistemas de aumentación SBAS (Satellite-based argumentation system), los 
ISSN: 2600-5859

datos recolectados fueron sometidos a un tratamiento de post corrección diferencial de posicionamiento (Jiménez \& Naranjo, 2009).

Los datos post corregidos fueron ingresados a una hoja de cálculo en coordenadas UTM (Universal Transverse Mercator), las cuales responden a un formato compatible con los softwares GIS (del inglés, Geographic Information System), para su posterior análisis (Montenegro \& Vargas, 2015). Con esta información y apoyados en el mapa base "Imagery with Labels" de ESRI (Environmental Systems Research Institute) se realizó la localización de las coordenadas, obteniendo como resultado el mapa de la Figura 1, que representa la ubicación geográfica del bosque "San Vicente".

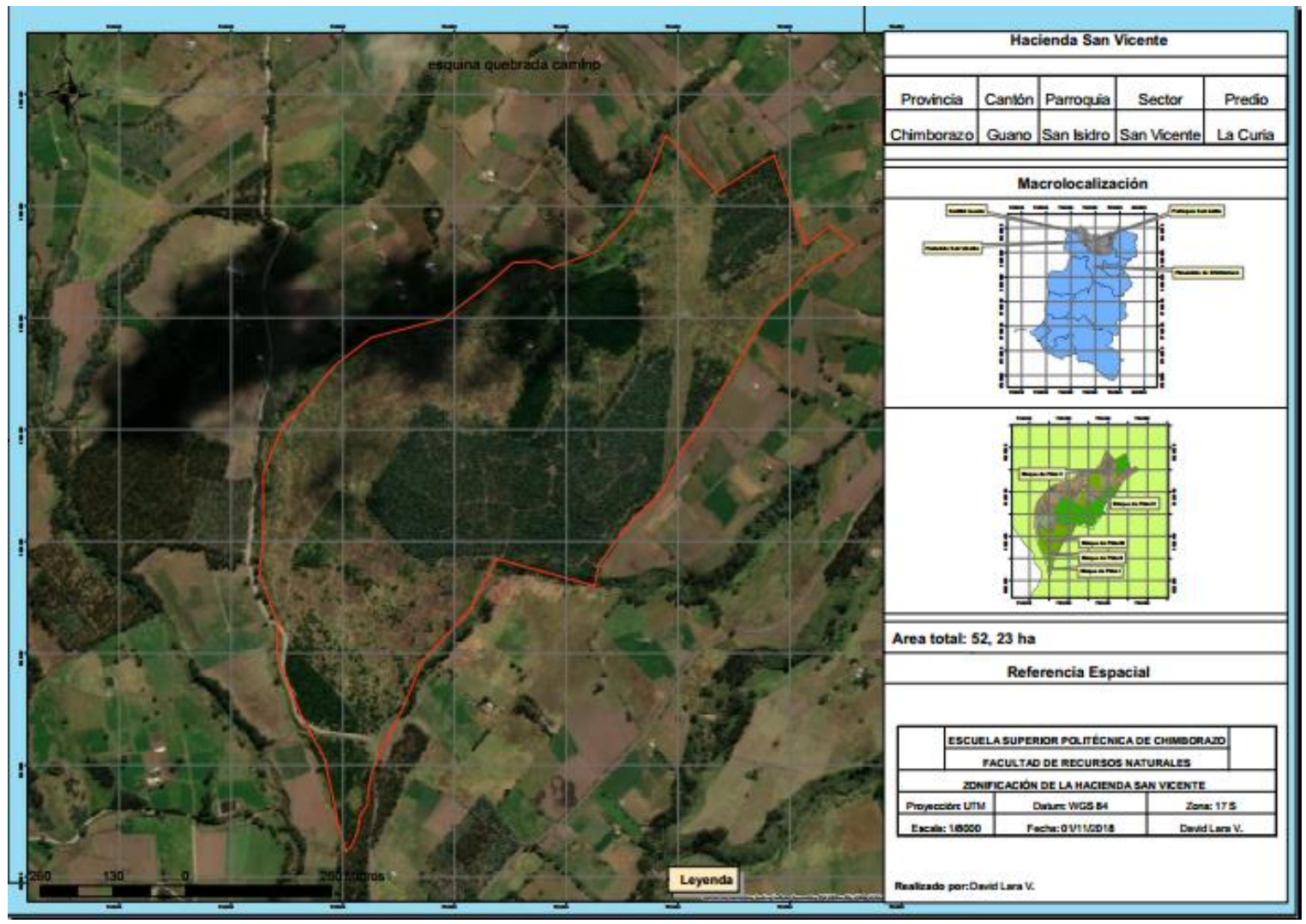

Figura 1. Mapa de la ubicación geográfica del bosque.

Fuente: Elaboración Propia.

\section{Caracterización de uso por tipo de área}

En base a un recorrido general del predio en estudio se identificaron 5 bloques y sus características. Igualmente se aplicó el muestreo sistemático, censo y muestreo exploratorio de acuerdo a lo especificado en la Tabla 1 en base a lo propuesto por la FAO (2011). 
ISSN: 2600-5859

Tabla 1. Tipos de muestreo utilizado por rodal evaluado

\begin{tabular}{cc}
\hline Áreas & Tipo de muestreo \\
\hline Bloque 1 & Muestreo sistemático 5\% \\
Bloque 2 & Censo \\
Bloque 3 & Muestreo exploratorio \\
Bloque 4 & Muestreo exploratorio \\
Bloque 5 & Muestreo exploratorio \\
\hline
\end{tabular}

En el caso del rodal 1, se realizó un muestreo piloto, y con la información preliminar se determinó el tamaño de la muestra utilizando la ecuación 1. Posteriormente se complementó mediante el muestreo sistemático a una intensidad de $4 \%$ con la instalación de parcelas circulares de $250 \mathrm{~m} 2$. Dentro de cada parcela se midió y registro el DAP (Diámetro a la Altura del Pecho) y la altura total promedio de los árboles, información con la cual se calculó la media muestral, el error de muestreo, los límites de confianza, la tabla de altura local, la tabla de volumen y el número de árboles por hectárea (Gautreau \& Lezama, 2009), mediante el uso de las ecuaciones que se indican a continuación:

El tamaño de la muestra se determinó con:

$$
\mathrm{n}=\frac{\mathrm{CV}^{2} * \mathrm{z}^{2}}{\mathrm{a} 2}
$$

Para calcular el volumen en pie de los individuos se utilizó la fórmula [2] (Ecuador \& Forestal, 2010).

$$
V=\frac{3.1416 \times(\text { Dap })^{2} \times h \times f}{4}
$$

Dónde: $\mathrm{V}=$ volumen total del árbol $\left(\mathrm{m}^{3}\right)$, Dap = Diámetro del árbol a la altura del pecho $(\mathrm{m}) ; \mathrm{h}=$ Altura comercial o total del árbol $(\mathrm{m}), \mathrm{ff}=$ factor de forma $=0.7$

Con los datos obtenidos del volumen se determinó la desviación estándar utilizando la fórmula:

$$
S=\sqrt{\frac{\sum X^{2}-\frac{\sum X^{2}}{n}}{n-1}}
$$


El error estándar se manejó a través de la expresión:

$$
S x=\frac{S}{\sqrt{n}}
$$

Para el cálculo del error de muestreo fue necesario hacer uso de la ecuación:

$$
E . M=\frac{s}{\sqrt{n}} * t
$$

Y finalmente para obtener el límite de confianza se aplicaron las expresiones:

$$
\begin{aligned}
& L s=x+E \cdot M \\
& L i=x-E \cdot M
\end{aligned}
$$

Cabe indicar que la variable altura fue estimada mediante la elaboración de la tabla de altura local con la fórmula:

$$
H=a+b(\operatorname{Ln} D A P)
$$

Donde $\mathrm{H}$ es la altura estimada de los árboles y DAP es el diámetro de los árboles; el coeficiente $a$ se calcula con la ecuación:

$$
a=y-b *(x)
$$

En tanto que b se obtiene con la siguiente ecuación:

$$
b=\frac{n \sum x y-\left(\sum x\right)\left(\sum y\right)}{n \sum x^{2}-\sum x^{2}}
$$

La tabla de volumen local se calcula con la fórmula:

$$
\mathrm{Ve}=\mathrm{a}+\mathrm{b}(\mathrm{Vr})_{[11]}
$$

Donde $\mathrm{Ve}$ es el volumen estimado, y $\mathrm{Vr}$ es el volumen real. 
ISSN: 2600-5859

El coeficiente de determinación usado para comprobar la calidad del modelo en la regresión lineal de la altura estimada entre las variables altura estimada y variable independiente se calculó en el software estadístico R (Risk \& Risk, 2003).

En el rodal 2 se midió el, altura total de todos los árboles, variables utilizadas para el cálculo del volumen de madera en pie por una parte y por otra la verificación de resultados con estimadores estadísticos.

Para los rodales 3 y 5 se aplicó un muestreo exploratorio mediante la observación directa y el registro de características generales.

En cambio, en el rodal 4, se realizó un muestreo exploratorio mediante la recolección de ejemplares botánicos, su herborización, el uso de bibliografía especializada, y el proceso culminó con la identificación de muestras botánicas.

Finalmente, con la información obtenida de los rasgos evaluados que presentó cada rodal se generaron estrategias silvícolas de manejo.

\section{Resultados}

\section{Descripción de la situación actual y cálculo de indicadores dasométricos del predio forestal} San Vicente.

Los resultados obtenidos indican la presencia de 5 rodales existentes en el predio forestal San Vicente, los cuales han sido clasificados de acuerdo al tipo de especies que los conforman, tal como se indica en la Tabla 2.

Tabla 2. Zonificación del área estudiada.

\begin{tabular}{ccccc}
\hline Rodal 1 & Rodal 2 & Rodal 3 & Rodal 4 & Rodal 5 \\
\hline Pinus radiata. & $\begin{array}{c}\text { Cupressus } \\
\text { macrocarpa. }\end{array}$ & $\begin{array}{c}\text { Eucalyptus } \\
\text { globulus }\end{array}$ & Zona de conservación & Zona \\
(Pino) & (Ciprés) & (Eucalipto) & $\begin{array}{c}\text { conformada por } \\
\text { especies vegetales }\end{array}$ & aprovechada \\
$\begin{array}{c}\text { 5 Bloques de } \\
\text { plantaciones }\end{array}$ & $\begin{array}{c}\text { 1 Bloque de } \\
\text { plantación }\end{array}$ & $\begin{array}{c}\text { 2 Bloques de } \\
\text { plantaciones }\end{array}$ & & \\
& & & & \\
\hline
\end{tabular}


ISSN: 2600-5859

Los 5 rodales existentes han sido sectorizados de acuerdo a sus componentes y representados en un mapa geográfico con diferente color, tal como se indica en la Figura 2, señalando la ubicación territorial del área estudiada.

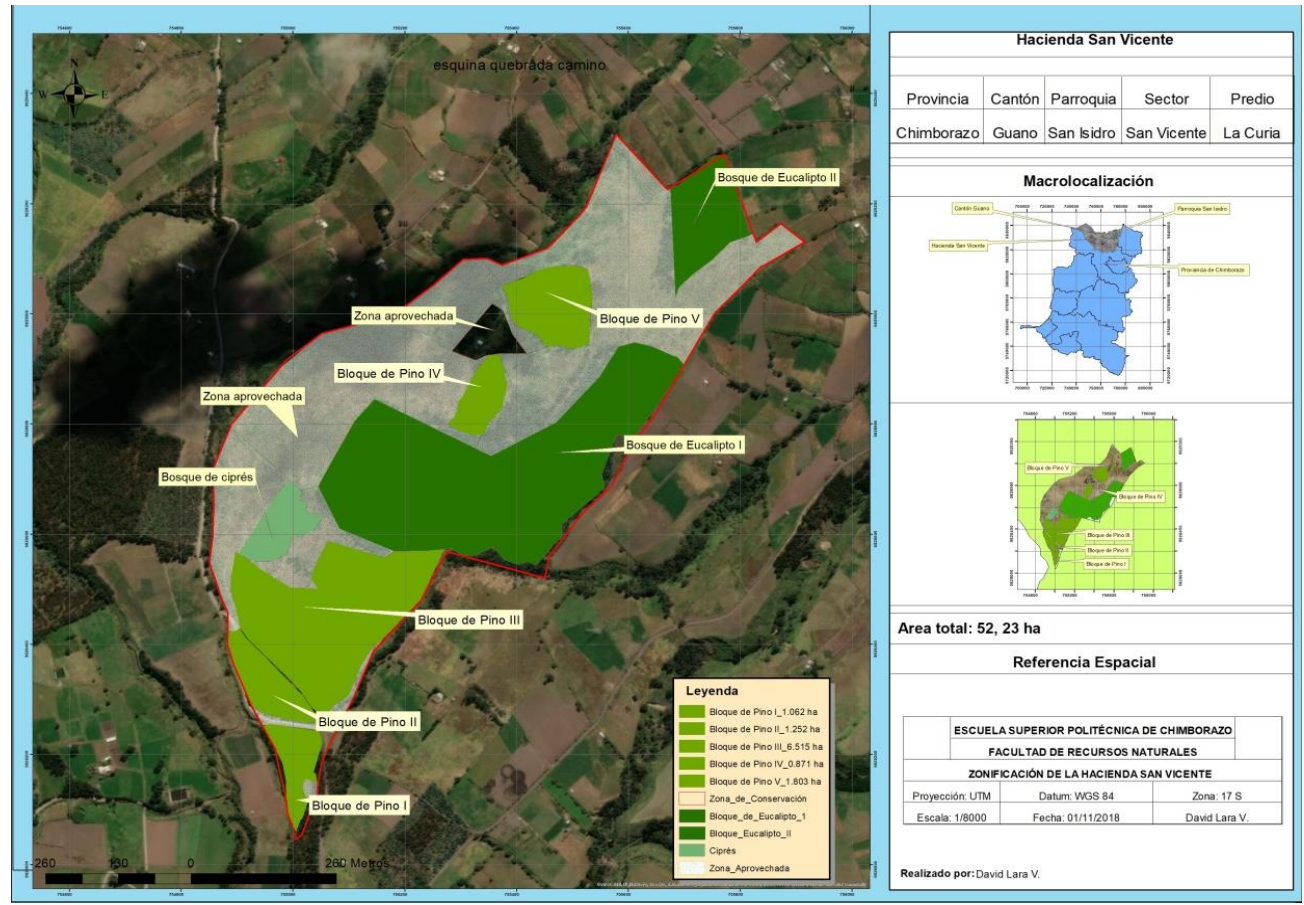

Figura 2. Mapa de rodalización del predio en el bosque.

Fuente: Elaboración Propia.

Rodal 1: Área de plantación de Pinus radiata. - Está conformada por 5 Bloques distribuidos en diferentes lugares geográficos de la hacienda, con altitudes que van desde los $3176 \mathrm{msnm}$ hasta alcanzar los $3286 \mathrm{msnm}$. Se caracterizan por ser coetáneos, presentando un diámetro medio de $16 \mathrm{~cm}$, mientras que su altura promedio es de $12,53 \mathrm{~m}$, con edades aproximadas de 8 años, tal como muestra la Tabla 3.

Tabla 3. Datos correspondientes a los 5 bloques que conforman el rodal uno de pino.

\begin{tabular}{ccccccccc}
\hline \multirow{2}{*}{$\begin{array}{c}\text { Blo- } \\
\text { ques }\end{array}$} & Altura & \multicolumn{2}{c}{ Ubicación } & $\begin{array}{c}\text { Exten- } \\
\text { sión }\end{array}$ & Edad & Diámetro & $\begin{array}{c}\text { Altura } \\
\text { aprox. }\end{array}$ & Volumen \\
\cline { 2 - 7 } & (m.s.n.m.) & $\mathrm{X}$ & $\mathrm{Y}$ & $\mathbf{( h a )}$ & $\mathbf{( a n ̃ o s )}$ & $\mathbf{( c m )}$ & $\mathbf{( m )}$ & $\left.\mathbf{( m}^{\mathbf{3}}\right)$ \\
\hline $1^{\circ}$ & 3176 & 760962 & 9814232 & 1,06 & 8 & $10-19.4$ & 11.54 & 156.66 \\
$2^{\circ}$ & 3185 & 755053 & 9828258 & 1.25 & 8 & $10-24.5$ & 12.48 & 201.43 \\
$3^{\circ}$ & 3185 & 755051 & 9828264 & 6,51 & 8 & $10-28.1$ & 12.96 & 767.92 \\
$4^{\circ}$ & 3239 & 755339 & 9828784 & 0.87 & 8 & $10-24.4$ & 14.94 & 161.69 \\
$5^{\circ}$ & 3286 & 755484 & 9828939 & 1,8 & 8 & $10-23$ & 12.26 & 356.88 \\
\hline
\end{tabular}


ISSN: 2600-5859

El valor obtenido para la variable altura de los árboles, es ligeramente inferior al valor de 12,76 m para árboles de 10 años de edad, reportado en condiciones de clima y suelo similares (Guallpa, \& Rosero, 2018).

El incremento medio anual para la altura estimado de 1,57 $\mathrm{m}$ es ligeramente superior en referencia al valor de 1,56 m año ${ }^{-1}$ a los 9 años de edad en Galicia (Sánchez \& Rodríguez, 2003). Asimismo, coincide con el 1,53 m año-1, incremento determinado en un rodal de la misma especie sometido a raleos y podas en la parroquia Mulaló (Paguanquiza, 2012).

Al ser el bosque de pino una especie de interés maderable se hizo un análisis que determina la cantidad volumétrica de madera que se podría obtener por cada bloque para el rodal uno. Se obtuvo como resultados el número de árboles y volumen promedio por ha, el valor de desviación estándar, coeficiente de variación y el número de parcelas requeridas por bloque evaluado (Tabla 4).

Tabla 4. Resultados del muestreo de los 5 bloques del rodal uno de Pinus radiata

\begin{tabular}{ccccccc}
\hline Sector & ha & $\begin{array}{c}\mathbf{N}^{\circ} \mathbf{d e} \\
\text { árboles /ha }\end{array}$ & $\begin{array}{c}\mathbf{X} \\
\left(\mathbf{m}^{\mathbf{3} / \mathbf{h a}}\right)\end{array}$ & $\mathbf{S}\left(\mathbf{m}^{\mathbf{3}} \mathbf{h} \mathbf{h a}\right)$ & $\mathbf{C V ~ \%}$ & $\mathbf{n}$ \\
\hline Bloque 1 & 1.062 & 396 & 150.3 & 21.37 & 14.22 & 4 \\
Bloque 2 & 1.252 & 395 & 173.78 & 25.20 & 14.50 & 4 \\
Bloque 3 & 6.515 & 320 & 128.88 & 10.02 & 7.70 & 3 \\
Bloque 4 & 0.871 & 626 & 183.85 & 22.16 & 12.05 & 3 \\
Bloque 5 & 1.803 & 350 & 123.10 & 9.90 & 8.04 & 3 \\
\hline
\end{tabular}

Los cálculos de volumen de los 5 rodales de pino arrojaron como resultado una superficie de análisis en cada bloque con un valor máximo de 6.515 ha y mínimo de 0.817 ha, así como también, los valores de altura local, tamaño de la muestra, media muestral, error de muestreo, límites de confianza, volumen de área basal, número de árboles por hectárea y relación diámetro altura.

La altura individual fue tomada en observación directa en el área de estudio proveniente de 51 árboles pertenecientes a 17 parcelas, mientras que la altura promedio estimada fue determinada por la ecuación logarítmica en donde " " toma un valor de 7,0437 y el valor del coeficiente "” es de 1,862, determinando así una altura promedio estimada de $12,756 \mathrm{~m}$.

La regresión lineal de la altura estimada manifiesta que posee el 100\%, indicando la relación existente entre las variables Altura Estimada e ; por su parte cuadrado posee un $100 \%$, lo que muestra que la variable Altura Estimada es explicada gracias a la variable independiente de igual manera con un cuadrado ajustado del $100 \%$ y un error estándar de $0 \%$, arrojando un coeficiente de determinación (Figura 3). 
ISSN: 2600-5859

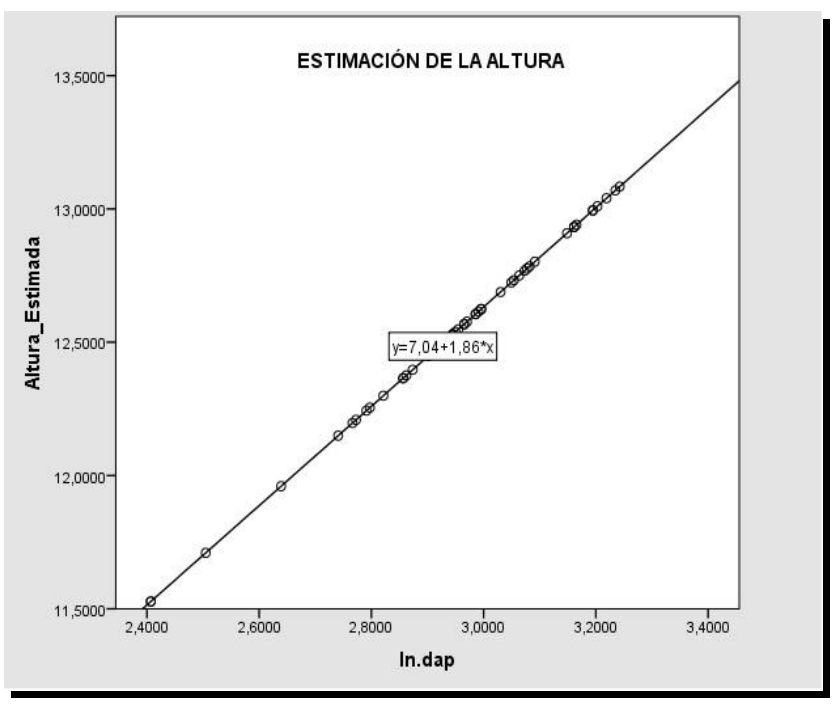

Figura 3. Regresión lineal aplicada a la estimación de altura. Variable dependiente: Altura Estimada. Predictores: (Constante),

Fuente: Elaboración Propia

Por su parte, la exactitud de la ecuación logarítmica de las alturas estimadas de la plantación de pino, indican una diferencia agregada ().

$$
\begin{gathered}
\mathrm{DA}=\frac{\sum \text { Altura } \mathrm{R}-\sum \text { Altura Est* } 100}{\sum \text { Altura Est }} \\
\mathrm{DA}=\frac{12,5445098-12,5445098 * 100}{12,5445098} \\
\text { DA }=0.00 \%
\end{gathered}
$$

Calculando la diferencia entre la altura real y la altura estimada. Otro indicador de exactitud es la Desviación media ()

$$
\begin{gathered}
\mathrm{DM}=\frac{\sum(\text { Altura } \mathrm{R}-\text { Altura Est }) / \mathrm{n}^{\star} 100}{\sum \text { Altura Est }} \\
\mathrm{DM}=\frac{7.3346^{\star} 100}{42} \\
\mathrm{DM}=15.79 \%
\end{gathered}
$$


En este caso la desviación media presenta un valor de $15.79 \%$.

Con el uso de variables combinadas se realizó la estimación de volumen mediante regresión lineal, en donde se obtuvieron las constantes a y b que fueron aplicadas en la fórmula:

$$
\begin{gathered}
Y=a+b(\text { DAP2 *H) } \\
Y=0,252+0,000000625 *(\text { DAP * } 2 * H) .
\end{gathered}
$$

La regresión lineal de la altura estimada manifiesta que $\mathrm{R}$ posee el 100\%, indicando la relación existente entre las variables Volumen_estimado y Dap2.h; por su parte R cuadrado posee un 100 $\%$, lo que muestra que la variable dependiente Dap2.h es explicada gracias a la variable independiente Volumen estimado, igual con un R cuadrado ajustado del 100\% y un error estándar de la estimación de $0 \%$ (Figura 4), logrando estimar un volumen total de $1644.58 \mathrm{~m}^{3}$ de madera de pino existente en la plantación de bosque en la hacienda.

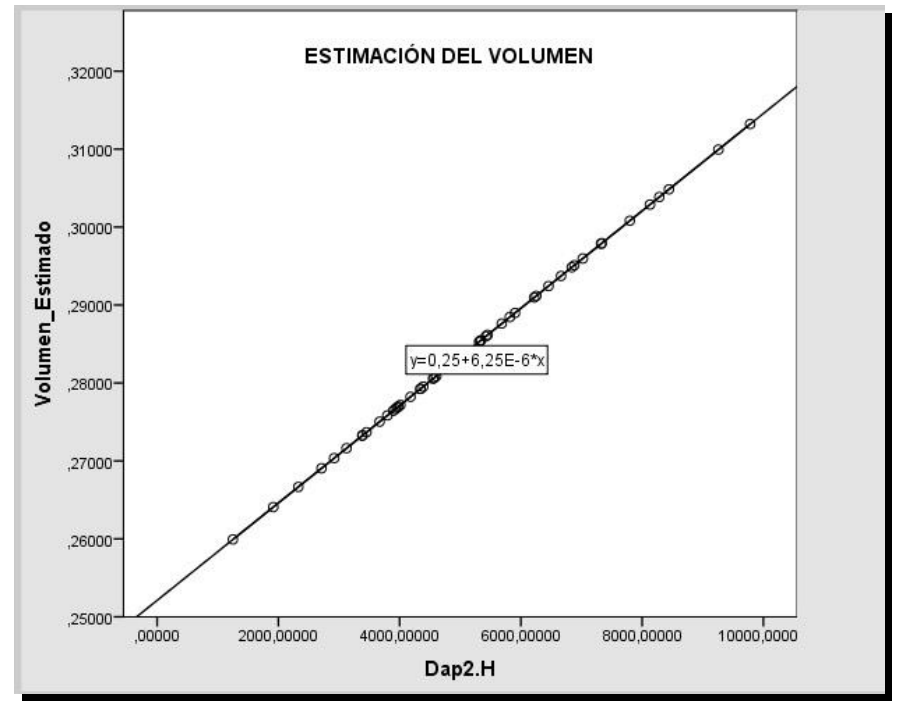

Figura 4. Regresión Lineal aplicada en la estimación de Volumen. Variable dependiente: . Predictores: (Constante), Volumen Estimado.

Fuente: Elaboración Propia

En cuanto al cálculo de exactitud que existe en los valores hallados de volumen calculado respecto al volumen real, se obtuvo tanto la diferencia agregada como la Desviación media, que es otro indicador de exactitud; hallándose los siguientes valores:

1. Diferencia agregada ()

$$
\mathrm{DA}=\frac{\sum \text { Volumen } \mathrm{R}-\Sigma \text { Volumen Est* } 100}{\sum \text { Volumen Est }}
$$




$$
\begin{gathered}
D A=\frac{14,50597-14,50598 * 100}{14,50598} \\
D A=-0,0000689 \%
\end{gathered}
$$

2. Desviación media (DM):

$$
\begin{gathered}
\mathrm{DM}=\frac{\sum(\text { Volumen } \mathrm{R}-\text { Volumen Est }) / \mathrm{n}^{*} 100}{\text { Volumen Est }} \\
\mathrm{DM}=9.42687578 * 100 \\
\mathrm{DM}=18,48 \%
\end{gathered}
$$

Rodal 2: Está conformado por una plantación de Cupressus macrocarpa, la cual se encuentra a 3191 msnm cubriendo una extensión de 1,040 ha, abarcando el 2,029 \% de la extensión total de la hacienda San Vicente. Esta zona se caracteriza por ser una plantación no manejada a la cual es difícil ingresar debido a la cercanía existente de árbol a árbol; posee una edad de 8 años, con un DAP de $10,8 \mathrm{~cm}$ a $16 \mathrm{~cm}$ y alturas aproximadas que alcanzan los 11,47 $\mathrm{m}$ con un volumen de aprovechamiento de 34,029 m3, el cual fue calculado mediante un censo realizado en 300 árboles de esta especie.

Rodal 3: Está conformado por dos bloques de Eucalyptus globulus, los cuales en su mayoría son rebrotes que no superan los $10 \mathrm{~cm}$ de DAP con una edad aproximada de tres años, es por eso que no fueron tomados en cuenta para el inventario de aprovechamiento comercial (Tabla 5).

Tabla 5. Distribución de plantación de Eucalyptus globulus

\begin{tabular}{clccccc}
\hline Bloques & $\begin{array}{l}\text { Altura } \\
\text { (m.s.n.m.) }\end{array}$ & \multicolumn{2}{c}{ Ubicación } & \multirow{2}{*}{$\begin{array}{c}\text { Extensión } \\
\text { (ha) }\end{array}$} & $\begin{array}{c}\text { Edad } \\
\text { (años) }\end{array}$ & $\begin{array}{c}\text { Porcentaje } \\
\text { respecto al área } \\
\text { total }\end{array}$ \\
\cline { 3 - 4 } & & & & & & \\
\hline $1^{\circ}$ & 3231 & 755646 & 9828940 & 12.246 & 3 & $24,77 \%$ \\
$2^{\circ}$ & 3331 & 755675 & 9829230 & 2,108 & 3 & $4 \%$ \\
\hline
\end{tabular}

\section{Rodal 4: Rodal de conservación}

Se encuentra alrededor de las casonas del predio San Vicente a una altitud de $3258 \mathrm{msnm}$ y cubre una extensión de 0.687 ha que representan el 1,30 \% de la hacienda evaluada. 
ISSN: 2600-5859

Este rodal está caracterizado por la presencia de especies arbóreas, arbustivas y herbáceas de interés ecológico, cuyo muestreo reportó un total de 18 individuos identificados pertenecientes a 14 familias, 18 géneros y 18 especies, correspondiendo las más frecuentes a la familia Asteraceae con 4 individuos como se muestra en la Tabla 6.

Tabla 6. Especies vegetales de interés ecológico identificadas.

\begin{tabular}{|c|c|c|c|c|}
\hline $\mathbf{N}^{\circ}$ & FAMILIA & GÉNERO & ESPECIE & N. VULGAR \\
\hline \multirow{4}{*}{1} & \multirow{4}{*}{ ASTERACEAE } & Baccharis & Baccharis latifolia (Ruiz \& P.) & Chilca \\
\hline & & Bidens & Bidens andicola $(\mathrm{L})$ & Cilantrillo \\
\hline & & Achyrocline & Achyrocline alata (Kunth) & Algodón \\
\hline & & Ageratina & Ageratina sp. (Spach) & $\begin{array}{l}\text { Maravilla de } \\
\text { monte }\end{array}$ \\
\hline 2 & APOCYNACEAE & Vinca & Vinca major $(\mathrm{L})$ & Hierba doncella \\
\hline 3 & AGAVACEAE & Yucca & Yucca guatemalensis (Baker) & Yuca gigante \\
\hline 4 & BETULACEAE & Alnus & Alnus acuminata (Kunth) & Aliso \\
\hline 5 & BIGNONIACEAE & Tecoma & Tecoma stans (Juss.) & Cholán \\
\hline 6 & FABACEAE & Genista & Genista monspessulana (L) L. A. S & Retama \\
\hline \multirow{2}{*}{7} & \multirow{2}{*}{ GERANACEAE } & Paraserianthes & Paraserianthes lophantha (Willd) & Pedo chino \\
\hline & & Geranium & Geranium laxicaule (Kunth) & Geranio \\
\hline 8 & POACEA & Stipa & Stipa ichu (Ruiz \& P.) & Paja \\
\hline 9 & POLYGALACEAE & Monnina & Monnina angustifolia (Kunth) & \\
\hline 10 & RUBIACEAE & $\begin{array}{l}\text { Arcytophy- } \\
\text { llum }\end{array}$ & Arcytophyllum thymifolium (Willd) & Morlan blanco \\
\hline 11 & ROSACEAE & Polylepis & Polylepis lanuginosa (Ruiz \& P.) & Árbol de papel \\
\hline 12 & $\begin{array}{l}\text { SCROPHULARIA- } \\
\text { CEAE }\end{array}$ & Buddleja & Buddleja incana (Ruiz \& P.) & Quishuar \\
\hline 13 & SOLANACEAE & Brugmansia & Brugmansia sanguinea (Ruiz \& P.) & Floripondio rojo \\
\hline \multirow{2}{*}{14} & \multirow{2}{*}{ SOLANACEAE } & \multirow{2}{*}{ Solanum } & Solanum nigrescens & \multirow{2}{*}{ Hierba mora } \\
\hline & & & (M. Martens \& Galeotti) & \\
\hline
\end{tabular}

\section{Rodal 5: Zona aprovechada}

El rodal 5, con una superficie de 24,67 ha, representa el 47,23\% de la totalidad del predio que ha sido intervenido, donde se observaron tocones de árboles de Pinus radiata cosechados mediante el método de corta a tala rasa (FONAM, 2007).

En cuanto a la zona aprovechada que se sitúa dentro del área de estudio, la información tomada y el posterior análisis de la misma indica que el bosque San Vicente tiene áreas de plantaciones forestales que ya han sido intervenidas y aprovechadas. 
ISSN: 2600-5859

\section{Plan de manejo del predio forestal San Vicente}

Una vez definidas las características de los rodales en estudio, se procedió a esquematizar las acciones a proponer en el plan de manejo de los mismos que se muestra a continuación en la Tabla 7.

Tabla 7. Esquema de manejo del predio forestal.

\begin{tabular}{cl}
\hline Rodales & \multicolumn{1}{c}{ Actividades silvícolas } \\
\hline 1 & $\begin{array}{l}\text { Raleo sistemático a una intensidad del 20\% complementado con poda a } \\
\text { los árboles remanentes. } \\
\text { Aplicar la actividad de poda a todos los árboles de Cupressus } \\
\text { macrocarpa }\end{array}$ \\
3 & Manejo de rebrotes dejando entre dos o tres , luego aplicar fertilización \\
4 & Monitoreo de la vegetación cada dos años \\
5 & $\begin{array}{l}\text { Reforestación con fertilización inicial con plántulas de Pinus radiata a } \\
\text { un distanciamiento de } 3 \times 3 \mathrm{~m}\end{array}$ \\
\hline
\end{tabular}

Se propone aplicar por una parte un raleo a una intensidad entre el 20 al 30\% para los bloques del rodal 1 dando una superficie total de 11,49 ha, a fin de que los árboles sobrantes luego del raleo optimicen de mejor manera su espacio de crecimiento en cada bloque con la finalidad de favorecer el crecimiento de los mismos. Por lo expuesto es importante aplicar el aprovechamiento y manejo de los bloques en mención, alternativa que coincide con lo sostenido por el INAB (Instituto Nacional de Bosques) (2013) el cual sugiere que la aplicación de raleo en la fase de crecimiento de un bosque plantado contribuye a redistribuir su crecimiento y productividad.

Una vez efectuado el raleo será importante complementar con la actividad de poda de los árboles remanentes (Musálem, 2006).

Con respecto al rodal 2 formado por árboles de Cupresus macrocarpa, dado su estado de crecimiento y desarrollo, se propone aplicar la actividad de poda a fin de estimular el aumento en su productividad y dejar los mejores árboles para la extracción final (INAB, 2008).

En el caso del rodal 3 formado por árrboles de Eucalyptus globulus en estado de latizal se debe ejecutar un manejo gradual de los mismos 2 . Considerando la elaboración de madera aserrada y postes se podrían dejar entre 1-3 rebrotes por tocón, y para combustible (leña) se podría dejar un 
número mayor a tres rebrotes en base a una planificación operativa, táctica y estratégica para el manejo silvicultural (Guallpa, Lara, Espinoza, Guilcapi, \& Fosado, 2019).

Para el rodal 4 tipificado como área de conservación, el funcionamiento es a nivel de condiciones de sitio y régimen (frecuencia e intensidad) de perturbaciones. A nivel de especies, el funcionamiento sucesional se explica a través de las estrategias de dispersión, regeneración, capacidad de establecimiento y el patrón de desarrollo de cada especie, al igual que a través de la dirección y velocidad con que ocurren los cambios en la vegetación en un espacio y tiempo determinados. Por lo mencionado, se dejará como está y que los factores del sitio incidan hasta un próximo monitoreo (entre 2-4 años), lo que permitirá inferir sobre cambios en la composición de especies, densidad y cobertura de la vegetación, riqueza y diversidad. Normalmente implican un reemplazo gradual y sucesivo de especies, incluyendo las del dosel, cuyo impacto del manejo forestal sobre la conservación de la diversidad vegetal está relacionado con la intensidad del manejo y temporalidad (Monárrez et al., 2018).

Finalmente, el rodal 5 se plantea reforestar con el uso de plántulas bien lignificadas de Pinus radiata de entre 20 a $30 \mathrm{~cm}$ de altura a un distanciamiento de $3 \mathrm{~m} \mathrm{x} 3 \mathrm{~m}$ a fin de lograr cierta recuperación de variables estructurales, funcionales. Con el tiempo, el crecimiento y desarrollo del arbolado modificará las condiciones del micro sitio del rodal y áreas circundantes al predio evaluado (Ventura, Plascencia, Hernández de la Rosa, Ángeles, \& Aldrete, 2017).

\section{Conclusiones.}

- La delimitación de áreas de uso del suelo, las características dasométricas y de flora de rodales que constituyen el predio forestal San Vicente sustentan su ordenación quedando definidos cinco rodales, el primero formado por cinco bloques de árboles de Pinus radiata, el segundo integrado por árboles de Cupressus macrocarpa, el tercero consta de dos bloques de Eucalyptus globulus en estado latizal bajo, el cuarto formado por especies arbóreas, arbustivas, herbáceas y un quinto rodal o área aprovechada.

- A fin de mejorar la productividad del rodal uno, es fundamental aplicar raleo con una intensidad del 20 al 30\% a todos los bloques que integran el rodal uno, luego complementar con la actividad de poda a los árboles sobrantes de cada bloque. Es necesario también podar a los árboles de Cupresus macrocarpa del rodal dos, y en el caso del rodal 3, este requiere un manejo gradual de los árboles de Eucalyptus globulus en estado de latizal bajo con fines de madera aserrada y postes se podría dejar entre 1-3 rebrotes por tocón, y para combustible (leña) un número mayor a tres. En referencia al rodal cuatro, se dejará como está y que los factores del sitio incidan hasta el próximo monitoreo entre 2-4 años.

- Finalmente, para el rodal cinco, se plantea reforestar mediante el uso de plántulas bien lignificadas de Pinus radiata de entre 20 a $30 \mathrm{~cm}$ de altura a un distanciamiento de $3 \mathrm{~m} \mathrm{x}$ $3 \mathrm{~m}$, que implique una preparación técnica del suelo que incluya fertilización inicial, esto con el objeto de renovar su productividad propiciando de esta forma la generación de beneficios ecológicos, económicos y sociales al aplicar criterios de manejo sustentable. 
ISSN: 2600-5859

\section{Referencias bibliográficas.}

Alvarado, D. \& Ospina, J. (2015). Spatial Distribution of Tropical Dry Forest in Valle Del Cauca, Colombia. Colombia. Acta Biol. Colomb, 2020 (33). Retrieved from https://doi.org/10.15446/abc.v20n2.46703

Calvache, J. (2016). La investigación científica como alternativa en la formación profesional. Colombia: CEPUN.

Castellanos Bolaños, J. F., Treviño Garza, E. J., Aguirre Calderón, O. A., Jiménez Pérez, J., Musalem Santiago, M., \& López Aguillón, R. (2008). Estructura de bosques de pino pátula bajo manejo en Ixtlán de Juárez, Oaxaca, México Forest structure of managed patula pine in Ixtlán de Juárez, 14(2), 51-63. Retrieved from http://www.scielo.org.mx/scielo.php?script=sci_arttext\&pid=S1405-04712008000200005

Collazo, M. A. G., Panizza, A., \& Paruelo, J. M. (2013). Ordenamiento territorial de bosques nativos: Resultados de la zonificación realizada por provincias del norte argentino. Ecología Austral, 23(2), 97-107. Retrieved from https://www.agro.uba.ar/noticias/sites/default/files/ot_de_bosques_nativos__agustina_collazo.pdf

Ecuador \& Forestal (2010). Instructivo de cubicación de madera para controles forestales en vias terrestres. 1-15. Retrieved from http://ecuadorforestal.org/wpcontent/uploads/2010/05/Instructivo-Cubicación-de-Madera.pdf

FAO (2011). Diseños de muestreo de las Evaluaciones Forestales Nacionales. Obtenido de http://www.fao.org/fileadmin/user_upload/national_forest_assessment/images/PDFs/Spa nish/KR2_ES_4_.pdf

Fondo Nacional del Ambiente. FONAM. (2007). Guía práctica para la instalación y manejo de plantaciones forestales. Lima, Perú.

Fernández, D. F. (2015). Gobierno Autónomo descentralizado San Isidro Patulú. Retrieved from http://app.sni.gob.ec/sni-

link/sni/PORTAL_SNI/data_sigad_plus/sigadplusdiagnostico/0660820080001_diagnosti co_14-10-2015_19-08-28.pdf

Gautreau, P. \& Lezama, F. (2009). Clasificación florística de los bosques y arbustales de las sierras del Uruguay. Ecología Austral, (19), 81-92. Retrieved from https://www.researchgate.net/publication/262711344_Clasificacion_floristica_de_los_bos ques_y_arbustales_de_las_sierras_del_Uruguay 
ISSN: 2600-5859

Graziano da Silva, J. (2018). Las vías forestales hacia el desarrollo sostenible de los bosques del mundo-Las vías forestales hacia el desarrollo sostenible. FAO, (978-92-5-130715-1), 153. Retrieved from http://www.fao.org/publications/es

Grijalva, J., Checa, X., Ramos, R., Barrera, P. \& Limongi, R. (2012). Situación de los Recursos Genéticos Forestales - Informe País Ecuador. Preparado por el Programa Nacional de Forestería del INIAP con aval del INIAP/FAO/MAE/MAGAP/MMRREE. Documento sometido a la Comisión Forestal de la FAO-Roma, para preparación del Primer Informe sobre el Estado de los Recursos Genéticos Forestales en el Mundo. 95 p.

Guallpa, M., \& Rosero, S. (2018). Evaluación dasométrica de una plantación de Pinus radiata D. Don en el sector San Andrés, Guano, Ecuador. European Scientific Journal, 14 (15), 7889.

Guallpa, M., Lara, N., Espinoza, M., Guilcapi, E., \& Fosado, O. (2019). Valoración cualitativa de una plantación de Eucalyptus globulus Labill en el sector de Licto, Riobamba, Ecuador. Polo del Conocimiento, 4 (4), 126-152.

Instituto Nacional de Bosques. INAB. (2008). INSTITUTO NACIONAL DE BOSQUES, 2008. Propuesta de procedimientos para el establecimiento y seguimiento de parcelas permanentes de medición forestal en plantaciones beneficiarias del PINFOR. Guatemala.

Instituto Nacional de Bosques. INAB. (2013). Crecimiento y Productividad de Plantaciones Forestales de Teca en Guatemala. Guatemala.

Jiménez, F. \& Naranjo, J. E. (2009). Nuevos requerimientos de precisión en el posicionamiento de vehículos para aplicaciones ADAS. DYNA-Ingeniería e Industria, 84(3). Retrieved from

http://search.ebscohost.com/login.aspx ?direct=true\&profile=ehost\&scope=site\&authtype $=$ crawler \&jrnl=00127361\&AN=38313047\&h=klz5Yer1WAvIv8gl\%2BorzgF90WDDu W50JBON\%2FKEGJBciTZBJfCuQohj8JatjMPGGcMqFPzSw8YiHnNhLwzu9jtQ\%3D $\% 3 \mathrm{D} \& \mathrm{crl}=\mathrm{c}$

Jiménez, J., Aguirre, O., \& Kramer, H. (2001). Análisis de la estructura horizontal y vertical en un ecosistema multicohortal de pino-encino en el norte de México. Forest Systems, 10(2), 355-366. Retrieved from https://www.researchgate.net/publication/28052703_Analisis_de_la_estructura_horizonta 1_y_vertical_en_un_ecosistema_multicohortal_de_pino-encino_en_el_norte_de_Mexico

Monárrez, J., Pérez, G., López, C., Márquez, M., \& González, M. (2018). Efecto del manejo forestal sobre algunos servicios ecosistémicos en los bosques templados de México. 
ISSN: 2600-5859

Madera y Bosques, 24(2), 1-16. Retrieved from
https://doi.org/10.21829/myb.2018.2421569

Montenegro, K. \& Vargas, D. (2015). Determinación de Puntos Geográficos Para el Cálculo de Áreas Exactas Utilizando Gps Diferencial Dgps. Retrieved from https://doi.org/10.20868/UPM.thesis.39079

Murrieta, E., Bryan, F., Delgado, D., Villalobos, R., \& Campos, J. (2007). Corredor Biológico Volcánica Central Identificación y caracterización. (51), 57-68. Retrieved from http://bco.catie.ac.cr/portal-revistas/index.php/RRNA/article/view/184

Musálem, M. (2006). Silvicultura de Plantaciones Forestales Comerciales. Chapingo, México: Universidad Autónoma Chapingo División de Ciencias Forestales

Ordóñez, S., Rivera Seria, E., Ordóñez, L., Espinosa, L., \& Rivera, P.M.B. (2004). La vegetación de los Andes del Ecuador. 1-28. Retrieved from http://www.flacsoandes.edu.ec/libros/digital/43572.pdf

Paguanquiza, E. (2012). Elaboración de una línea base para determinar el crecimiento y desarrollo de las plantaciones de Pinus patula y Pinus radiata en la hacienda San Joaquin de Aglomerados Cotopaxi S.A (ACOSA)(Trabajo de pregrado). Escuela Superior Politécnica de Chimborazo, Chimborazo, Ecuador.

Risk, I. \& Risk, M. (2003). Cartas sobre Estadística de la Revista Argentina de Bioingeniería. In Revista chilena de obstetricia y ginecolog (Vol. 68). https://doi.org/10.4067/S071775262003000400017

Sánchez, F. \& Rodríguez, R. (2003). Selvicultura de Pinus radiata. Compostela: Universidad de Santiago de Compostela. 26

Sánchez, M. (2015). Ecuador: Revisión a las principales características del recurso forestal y de la deforestación. Revista Científica y Tecnológica UPSE, 3(1), 41. https://doi.org/10.26423/rctu.v3i1.70

Torres, O. \& Peña, F. (2011). Zonificación del potencial energético de la biomasa residual forestal en la cuenca del lago Ranco, Chile: Antecedentes para la planificación energética regional. Bosque (Valdivia), 32(1), 77-84. https://doi.org/10.4067/S0717-92002011000100009

Ventura, A., Plascencia, F., Hernández de la Rosa, P., Ángeles, G., \& Aldrete, A. (2019). ¿Es la reforestación una estrategia para la rehabilitación de bosques de pino? Una experiencia en el centro de México. Bosque, 38 (1), 55-56

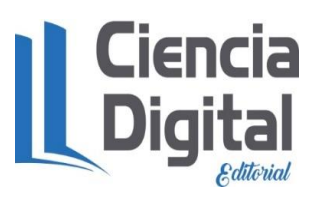

Creatividad, Diseño e Innovación

Página 166 
ISSN: 2600-5859

\section{Para citar el artículo indexado}

Chamorro Sevilla, H. E., Urquizo Buenaño, G. J., Cushpa Guamán, A. L., \& Lara Vásconez, D. F. (2020). Evaluación del predio forestal San Vicente con fines de manejo y conservación ubicado en el cantón guano, Ecuador. ConcienciaDigital, 3(1.1), 147-167. https://doi.org/10.33262/concienciadigital.v3i1.1.1137

El artículo que se publica es de exclusiva responsabilidad de los autores y no necesariamente reflejan el pensamiento de la Revista Conciencia Digital.

El articulo queda en propiedad de la revista y, por tanto, su publicación parcial y/o total en otro medio tiene que ser autorizado por el director de la Revista Conciencia Digital.
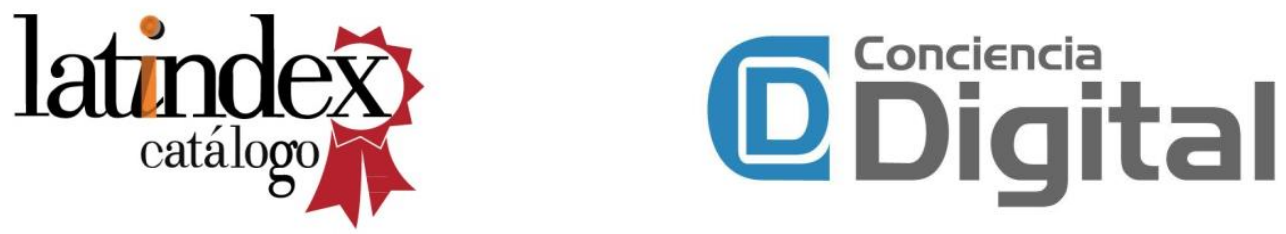